Original article

\title{
Evaluation of antibiotic consumption and compliance to hospital antibiotic policy in the surgery, orthopedics and gynecology wards of a tertiary care hospital
}

\author{
Aksa Panickal Thomas ${ }^{\mathrm{a}}$, Mahadevan Kumar ${ }^{\mathrm{b}}$, Roshna Johnson ${ }^{\mathrm{a}}$, Sneha Prakash More ${ }^{\mathrm{a}}$, \\ Bijoy Kumar Panda ${ }^{a, *}$
}

${ }^{a}$ Department of Clinical Pharmacy, Poona College of Pharmacy, Bharati Vidyapeeth (Deemed to be University), Pune, Maharashtra, India

${ }^{\mathrm{b}}$ Department of Microbiology, Bharati Vidyapeeth (Deemed to be University) Medical College and Hospital, Pune, Maharashtra, India

\section{A R T I C L E I N F O}

\section{Keywords:}

Antibiotics

Consumption

Compliance

Policy

\begin{abstract}
A B S T R A C T
Objectives: To evaluate antibiotic consumption and compliance to hospital antibiotic policy in the surgery, orthopedics and gynecology wards of a tertiary care hospital.

Methods: A prospective observational study was conducted over eight months on adult inpatients prescribed with antibiotics. Data were collected using a predesigned antibiotic form and evaluated using Defined Daily Dose per 100 bed-days. Antibiotics prescribed were classified using World Health Organization Access, Watch and Reserve classification (WHO AWaRe) and assessed for compliance to the hospital antibiotic policy which is in accordance with the National Treatment Guidelines for Antimicrobial use in Infectious Diseases (version 1.0.2016).

Results: Out of 2653 patients enrolled, $44.3 \%$ were males and 55.7\% were females. The antibiotic usage rate was $29 \%$. Cephalosporins were the most frequently prescribed class of antibiotics. The top three antibiotics prescribed were cefuroxime, metronidazole, and ceftriaxone. Classification of antibiotics using WHO AWaRe policy showed that $36 \%$ of antibiotics were prescribed from the Access group and $53 \%$ from the Watch group. The use of antibiotic combinations from the not recommended group of AWaRe was also observed. Total compliance observed towards the hospital's antibiotic policy was $77.7 \%$. The most common reason for non-compliance to hospital policy was inappropriate duration of antibiotic therapy.

Conclusions: The use of watch group of antibiotics over access group due to concerns of antimicrobial resistance shows the need for local adaptation of WHO AWaRe classification. Despite good adherence to the hospital's antibiotic policy, extended duration of antibiotic therapy requires special attention. Further optimization of antibiotic use can be achieved by timely revision and implementation of hospital antibiotic policy.
\end{abstract}

\section{Introduction}

India is among the largest consumers of antibiotics worldwide. ${ }^{1}$ Antibiotic sales data in India from 2000 to 2015 revealed that consumption of Watch and Reserve group antibiotics was increasing faster than consumption of Access group antibiotics. ${ }^{2}$ Studies have well documented the correlation between the quantities of antimicrobial agents used and the rate of development of antimicrobial resistance (AMR). ${ }^{3,4}$

Defined daily dose (DDD), drug utilization 90\% (DU90\%), World
Health Organization Access, Watch and Reserve classification (WHO AWaRe) policy and hospital antibiotic policy are tools to optimize the use of antibiotics in healthcare institutions. A recent meta-analysis of 89 studies assessed the effectiveness of antibiotic stewardship programs. Policy interventions changed antibiotic treatment and this was associated with significant improvement in outcomes. Unfortunately, there were hardly any studies from low and middle income countries in this meta-analysis. ${ }^{3}$ This is an important aspect to consider since infections and irrational antibiotic use are widely prevalent in these countries. Only few Indian studies have focused on the pattern and amount of

* Corresponding author. Department of Clinical Pharmacy, Poona College of Pharmacy, Bharati Vidyapeeth (Deemed to be University), Pune, Maharashtra, 411038, India.

E-mail addresses: aksathomas077@gmail.com (A.P. Thomas), dr.mkumarmicro@outlook.com (M. Kumar), roshnajohnson024@gmail.com (R. Johnson), Sneham1213@gmail.com (S.P. More), bijoy.panda@bharatividyapeeth.edu (B.K. Panda). 
antibiotic use as well as on the level of adherence to antibiotic usage guidelines in various hospital wards to improve prescribing behavior and control irrational use of antibiotics. ${ }^{5,6}$

Hence, the aim of this study was to assess antibiotic consumption in our hospital on the basis of volume using DDD and ranking antibiotic usage according to DU90\%. The proportion of antibiotics prescribed according to the WHO AWaRe classification and compliance to hospital antibiotic policy were also determined. The present study aims to bridge the gap and determine whether antibiotic policies are truly effective.

\section{Methods}

\subsection{Study setting}

This study was carried out in the surgery, orthopedics and gynecology wards of a tertiary care teaching hospital in western India. The antibiotic prescriptions of each ward were vetted by the clinical pharmacists and microbiologist under the antimicrobial stewardship program. The program formulates the Antibiotic Policy based on the antibiogram, hospital antibiotic formulary (published by the Drugs and Therapeutics Committee) and is tasked with implementation of antibiotic policy. The hospital antibiotic policy (version 5.0 .2019$)^{7}$ was adopted in all the wards.

\subsection{Study design and outcome variables}

A descriptive, prospective, cross-sectional study design was employed. Data was collected from inpatients case files over a period of eight months from July 2019 to February 2020. All the patients $(\geq 18$ years of age) receiving antibiotic treatment were included in the study, regardless of the indication. Sample size was determined using the following formula ${ }^{8}$ :

$n=\frac{Z^{2} p(1-p)}{d^{2}}=\frac{(1.96)^{2} \times 0.5(1-0.5)}{(0.025)^{2}}=1537$

Where: $\mathrm{n}=$ sample size.

$\mathrm{P}=$ the estimate of the proportion of patients with antibiotics $=0.5$ (Due to a lack of previous studies, p was set to 0.5 to obtain the largest sample size possible.)

$\mathrm{d}=$ margin of sampling error tolerated $=0.025(2.5 \%)$

$\mathrm{Z}=$ the standard normal value at confidence interval of $95 \%=1.96$.

The study was approved by the institutional ethics committee (IEC) of the hospital. Waiver of patient consent was obtained from the IEC as the study did not require direct contact with patients. Physicians' orders and medication charts were checked from individual patient files. Parameters such as patient demographics, diagnosis, antibiotics, dose, reason for antibiotic use (prophylaxis, empirical, definitive), and laboratory parameters including hematological, biochemical and microbiological culture sensitivity results of each patient receiving antimicrobial treatment were recorded.

\subsection{Antibiotic utilization}

Defined daily dose (DDD) and drug utilization index (DU90\%) were the metrics used for quantifying antibiotic use. The dose and duration of prescribed antibiotics were collected from patients' case files. Consumption was calculated as DDD normalized for 100 bed-days. ${ }^{9}$ DDD per 100 bed-days is an important indicator of inpatient antibiotic use.

$\mathrm{DDD} / 100$ bed-days $=$ (consumption of antibiotics during study period $[\mathrm{g}] \times$ 100)/ (DDD coefficient $[\mathrm{g}] \times$ total bed-days)

To assess the quality of drug utilization, antibiotics were ranked according to the volume of DDDs prescribed. Those antibiotics that accounted for $90 \%$ of the total volume, i.e. the DU90\%, were identified and compared within the wards. ${ }^{10}$

\subsection{Compliance to hospital antibiotic policy}

Hospital antibiotic policy version 5.0.2019 ${ }^{7}$ of our hospital was used to evaluate compliance of antibiotic prescribing in terms of indication, dosage, frequency and duration of treatment. Total compliance was defined as justifiably indicated antibiotic fulfilling the correct choice, dosage, frequency and duration of therapy. Non-compliance was considered when there was prescription of a non-indicated antibiotic, divergence from the advisable antibiotic or failure to have complete adherence with other aspects of therapy like dosage, frequency and duration of therapy.

\subsection{Data analysis}

Microsoft Excel 2016 was used for data entry and analysis. Proportions and means were calculated using descriptive analysis. Data was analyzed using Analysis of Variance (ANOVA). Significance was considered at $\mathrm{p}$-value $<0.05$.

\section{Results}

A total of 2653 (29\%) patients who received antibiotics were included in the study out of 9148 patients admitted during the study period. The rate of antibiotic use in the surgery and gynecology ward was $29.7 \%(1396 / 4700)$ and $25.1 \%(823 / 3274)$ respectively. A higher rate of antibiotic use was found in the orthopedics ward 36.9\% (434/ 1174). Antibiotics were mostly prescribed to men in surgery (63.3\%) and orthopedic (67.3\%) wards. Overall antibiotics were prescribed on average 1.8 times per patient. Antibiotics were prescribed as empiric therapy in $657(24.8 \%)$ patients, definitive therapy in $221(8.3 \%)$ patients, and surgical prophylaxis in 1775 (66.9\%) patients. Among the patients who received antibiotics for surgical prophylaxis, $400(22.5 \%)$ patients were treated for more than $24 \mathrm{~h} 360$ (54.7\%) of the patients prescribed antibiotics as empiric therapy, were treated for more than 3 days. A significant difference $(\mathrm{p}=0.005)$ was observed between the LOS of patients who received 1, 2 and 3 or more antibiotics as empirical therapy (Table 1).

Bacteriological investigations were performed on 570 (21.4\%) patients, with $309 / 570(54.2 \%)$ patients showing bacterial growth and $261 / 570$ (45.8\%) patients showing no bacterial growth. In this study, antibiotic therapy was escalated or de-escalated based on culture sensitivity reports in 364/570 (63.9\%) patients with positive cultures. Empiric antibiotic therapy was not de-escalated after receiving culture reports in 206/570 (36.1\%) patients (Table 2).

The overall systemic antibiotic use in the surgery, orthopedics, and gynecology wards was $31.85,35.7$, and 23.02 DDD/100 bed-days, respectively, based on defined daily dose (Table 3). A total of 21-34 systemic antibiotic substances were used; additionally, there were 5-13 different substances within the DU90\% segment as shown in Fig. 1. Antibiotics prescribed in the DU90\% segment accounted for $23.8-44.8 \%$ of total antibiotics prescribed. The survey also reveals significant variation in the pattern and amount of antibiotics used in each ward (Table 2; Fig. 1). Cefuroxime (6.4 DDD/100 BD), ceftriaxone (5.6 DDD/ $100 \mathrm{BD}$ ), metronidazole (4.5 DDD/100 BD), and amoxicillin-clavulanate (3.9 DDD/100 BD) were the most commonly used antibiotics in the surgery ward, ranking first, second, third, and fourth in terms of utilization. Cefuroxime (12 DDD/100 BD), amikacin (4.7 DDD/100 BD), ceftriaxone (4.6 DDD/100 BD), and metronidazole (3.5 DDD/100 BD) were the four most commonly used antibiotics in the orthopedics ward. Whereas, cefuroxime (11.2 DDD/100 BD), metronidazole (7.2 DDD/100 $\mathrm{BD})$, ceftriaxone (1.1 DDD/100 BD), and amoxicillin-clavulanate (0.9 $\mathrm{DDD} / 100 \mathrm{BD}$ ) were the most commonly used antibiotics in the gynecology ward (Fig. 1). 
Table 1

Demographic characteristics of patients.

\begin{tabular}{|c|c|c|c|c|}
\hline & Surgery & Orthopedics & Gynecology & Total \\
\hline $\begin{array}{l}\text { Total patients admitted } \\
\text { (9148) }\end{array}$ & 4700 & 1174 & 3274 & 9148 \\
\hline $\begin{array}{l}\text { Patients on antibiotics } \\
\quad(2653,29 \%)\end{array}$ & $\begin{array}{l}1396 \\
(29.7 \%)\end{array}$ & $\begin{array}{l}434 \\
(36.9 \%)\end{array}$ & $\begin{array}{l}823 \\
(25.1 \%)\end{array}$ & $\begin{array}{l}2653 \\
(29 \%)\end{array}$ \\
\hline \multicolumn{5}{|c|}{ Sex distribution of patients on antibiotics } \\
\hline Males & $\begin{array}{l}883 \\
(63.3 \%)\end{array}$ & $\begin{array}{l}292 \\
(67.3 \%)\end{array}$ & - & $\begin{array}{l}1175 \\
(44.3 \%)\end{array}$ \\
\hline Females & $\begin{array}{l}513 \\
(36.7 \%)\end{array}$ & $\begin{array}{l}142 \\
(32.7 \%)\end{array}$ & $823(100 \%)$ & $\begin{array}{l}1478 \\
(55.7 \%)\end{array}$ \\
\hline $\begin{array}{l}\text { Average number of } \\
\text { antibiotics per } \\
\text { encounter }\end{array}$ & 1.8 & 1.9 & 1.8 & 1.8 \\
\hline \multicolumn{5}{|c|}{ Type of antibiotic therapy $n=2653$} \\
\hline Empiric & $\begin{array}{l}403 \\
(28.9 \%)\end{array}$ & $49(11.3 \%)$ & $\begin{array}{l}205 \\
(24.9 \%)\end{array}$ & $\begin{array}{l}657 \\
(24.8 \%)\end{array}$ \\
\hline Definitive & $\begin{array}{l}141 \\
(10.1 \%)\end{array}$ & $44(10.1 \%)$ & $36(4.4 \%)$ & $\begin{array}{l}221 \\
(8.3 \%)\end{array}$ \\
\hline Prophylactic & $\begin{array}{l}852 \\
(61 \%) \\
\end{array}$ & $\begin{array}{l}341 \\
(78.6 \%) \\
\end{array}$ & $\begin{array}{l}582 \\
(70.7 \%) \\
\end{array}$ & $\begin{array}{l}1775 \\
(66.9 \%) \\
\end{array}$ \\
\hline Patients with SP $>24 \mathrm{~h}$ & $\begin{array}{l}295 \\
(34.6 \%)\end{array}$ & $35(10.2 \%)$ & $70(12 \%)$ & $\begin{array}{l}400 \\
(22.5 \%)\end{array}$ \\
\hline $\begin{array}{l}\text { Patients with }>\text { 3days } \\
\text { empiric therapy }\end{array}$ & $\begin{array}{l}290 \\
(71.9 \%)\end{array}$ & $35(71.4 \%)$ & $35(17 \%)$ & $\begin{array}{l}360 \\
(54.7 \%)\end{array}$ \\
\hline \multicolumn{5}{|c|}{ LOS of patients receiving empiric therapy (mean $\pm S D$ ) } \\
\hline 1 AMA & $6.2 \pm 4.3$ & $7.6 \pm 4.3$ & $6.7 \pm 4.7$ & $6.8 \pm 4.3$ \\
\hline 2 AMA & $8.7 \pm 8.5$ & $9.4 \pm 8$ & $9.1 \pm 2.6$ & $8.9 \pm 7.1$ \\
\hline$\geq 3$ AMAs & $\begin{array}{l}10.4 \pm \\
7.6\end{array}$ & $11.6 \pm 9.1$ & $9.7 \pm 4.3$ & $\begin{array}{l}10.4 \pm \\
7.4\end{array}$ \\
\hline P-value & 0.007 & 0.01 & 0.1 & 0.005 \\
\hline
\end{tabular}

*LoS- length of stay, *AMA- Antimicrobial Agent, *SP- Surgical Prophylaxis, *SD-Standard Deviation, *P-value was calculated using One way ANOVA Significant $(\mathrm{P}<0.05)$.

Table 2

Bacteriological Cultures $n(\%) ; n=$ number of patients $(570 / 2653)$.

\begin{tabular}{ll}
\hline Total patients & $570(21.4 \%)$ \\
Positive cultures & $309(54.2 \%)$ \\
No microbial growth & $261(45.8 \%)$ \\
Antibiotic Escalation/de-escalation performed & $364(63.9 \%)$ \\
Antibiotic De-escalation not performed & $206(36.1 \%)$ \\
\hline
\end{tabular}

The use of antibiotics in accordance with WHO AWaRe policy was also documented. Throughout the study period, 42 antibiotic agents (Access 10, Watch 22, Reserve 6, and Not recommended 4) were consumed. Out of 42 antibiotics consumed, 18 antibiotics (Access 6, Watch 8, Reserve 3, and Not recommended 1) accounted for DU $90 \%$ (Fig. 1). In the surgery and orthopedics wards, 13 antibiotics were observed in the DU 90\% segment, while in the gynecology ward, 5 antibiotics were observed in the DU $90 \%$ segment. In the orthopedic and gynecology wards, no reserve antibiotics were observed in the DU $90 \%$. In the surgery ward, reserve antibiotics (tigecycline, polymixin B and linezolid) accounted for $11.5 \%$ of antibiotics use in the DU $90 \%$ segment. The use of a Not-recommended antibiotic from the AWaRe policy (cefoperazone-sulbactam) was observed in the DU 90\% segment of surgery and orthopedic wards (Fig. 1). Not-recommended fixed-dose combination antibiotics, namely combinations of ciprofloxacintinidazole, ofloxacin-ornidazole, cefoperazone-sulbactam, and ceftriaxone-sulbactam, were also prescribed.

Out of 4871 prescribed antibiotics, 3783 (77.7\%) were in compliance with all aspects of the hospital's antibiotic policy. Non-compliance with the hospital antibiotic policy ${ }^{[9]}$ was attributed to incorrect duration of surgical prophylaxis in $725(14.8 \%)$ prescriptions, incorrect indication in 347 (7.1\%), incorrect dose/route and incorrect antibiotic choice in 8 $(0.2 \%)$ of each prescription as shown in Table 4 .

\section{Discussion}

In our study, the overall rate of antibiotic use was $29 \%$ which is similar to the antibiotic usage rate in surgical wards of western European countries (28\%). ${ }^{11}$ However, in a multicenter study conducted in India, ${ }^{12}$ the antibiotic usage rate was $57.4 \%$. Similarly, in western and central Asia and North America, it was observed to be $44.7 \%$ and $44.2 \%$, respectively. ${ }^{11}$ This was due to the real or perceived high prevalence of antibiotic resistance, absence or non-adherence to antibiotic treatment guidelines. Prophylactic antibiotics were given to $66.9 \%$ of patients (surgical). Cefuroxime (62.1\%) and metronidazole (26.2\%) were the most commonly prescribed antimicrobials for surgical prophylaxis (SP). In contrast, the most commonly prescribed antibiotics for prophylaxis (surgical) in a multicenter study (India) were cefuroxime (36\%), amikacin $(10 \%)$, and ceftriaxone $(8 \%) .{ }^{12}$ However, cefazolin was preferred globally as prophylaxis ${ }^{11}$ as it provides adequate coverage against the majority of organisms that cause postoperative infections, has fewer adverse effects, achieves optimal tissue levels, and is relatively inexpensive. ${ }^{13}$ Yet, cefuroxime was used in our hospital due to a lack of

Table 3

Antibiotic consumption in DDD/100 bed days.

\begin{tabular}{|c|c|c|c|c|c|c|c|c|}
\hline \multirow[t]{2}{*}{ Antibiotics } & \multicolumn{2}{|l|}{ Surgery } & \multicolumn{2}{|l|}{ Orthopedics } & \multicolumn{2}{|l|}{ Gynecology } & \multirow[t]{2}{*}{ Total DDD/100 BD } & \multirow[t]{2}{*}{ Total \% } \\
\hline & $\mathrm{DDD} / 100 \mathrm{BD}$ & $\%$ & DDD/100 BD & $\%$ & $\mathrm{DDD} / 100 \mathrm{BD}$ & $\%$ & & \\
\hline 1st generation cephalosporins & 0.004 & 0.01 & 0.3 & 0.8 & 0.1 & 0.4 & 0.09 & 0.3 \\
\hline 2nd generation cephalosporins & 6.4 & 20.1 & 12 & 33.6 & 11.2 & 48.7 & 8.7 & 29 \\
\hline 3rd generation cephalosporins & 7.1 & 22.3 & 6.1 & 17.1 & 1.3 & 5.6 & 5.3 & 17.6 \\
\hline 4th generation cephalosporins & & & 0.2 & 0.5 & & & 0.04 & 0.1 \\
\hline Aminoglycosides & 0.8 & 2.5 & 4.8 & 13.4 & 0.1 & 0.4 & 1.2 & 4 \\
\hline Quinolones & 1.5 & 4.7 & 0.7 & 2 & 0.2 & 0.9 & 1 & 3.3 \\
\hline Lincosamide & 0.8 & 2.5 & 0.3 & 0.9 & 0.01 & 0.05 & 0.5 & 1.6 \\
\hline Macrolide & 0.4 & 1.3 & 0.7 & 2 & 0.5 & 2.2 & 0.5 & 1.7 \\
\hline Nitroimidazole & 4.5 & 14.1 & 3.5 & 9.8 & 7.2 & 31.2 & 5.1 & 17 \\
\hline Nitrofurantoin & 0.1 & 0.3 & 0.2 & 0.6 & 0.2 & 0.9 & 0.1 & 0.3 \\
\hline Penicillins & 5.5 & 17.3 & 3.5 & 9.8 & 1.2 & 5.2 & 4 & 13.3 \\
\hline Tetracyclines & 0.2 & 0.6 & 0.7 & 2 & 0.5 & 2.2 & 0.3 & 1 \\
\hline Polymixins & 1.6 & 5 & 0.3 & 0.8 & & & 1 & 3.3 \\
\hline Carbapenems & 0.6 & 1.9 & 0.7 & 2 & 0.01 & 0.05 & 0.5 & 1.7 \\
\hline Linezolid & 0.6 & 1.9 & 0.3 & 0.8 & 0.5 & 2.2 & 0.5 & 1.7 \\
\hline Glycopeptides & 0.3 & 1 & 1.3 & 3.6 & & & 0.4 & 1.3 \\
\hline Tigecycline & 1.4 & 4.4 & 0.1 & 0.3 & & & 0.8 & 2.7 \\
\hline Fosfomycin & 0.05 & 0.1 & 0.01 & 0.02 & & & 0.03 & 0.1 \\
\hline Total & 31.854 & 100 & 35.7 & 100 & 23.02 & 100 & 30.06 & 100 \\
\hline
\end{tabular}

*DDD- Defined daily dose, *BD - Bed Days. 


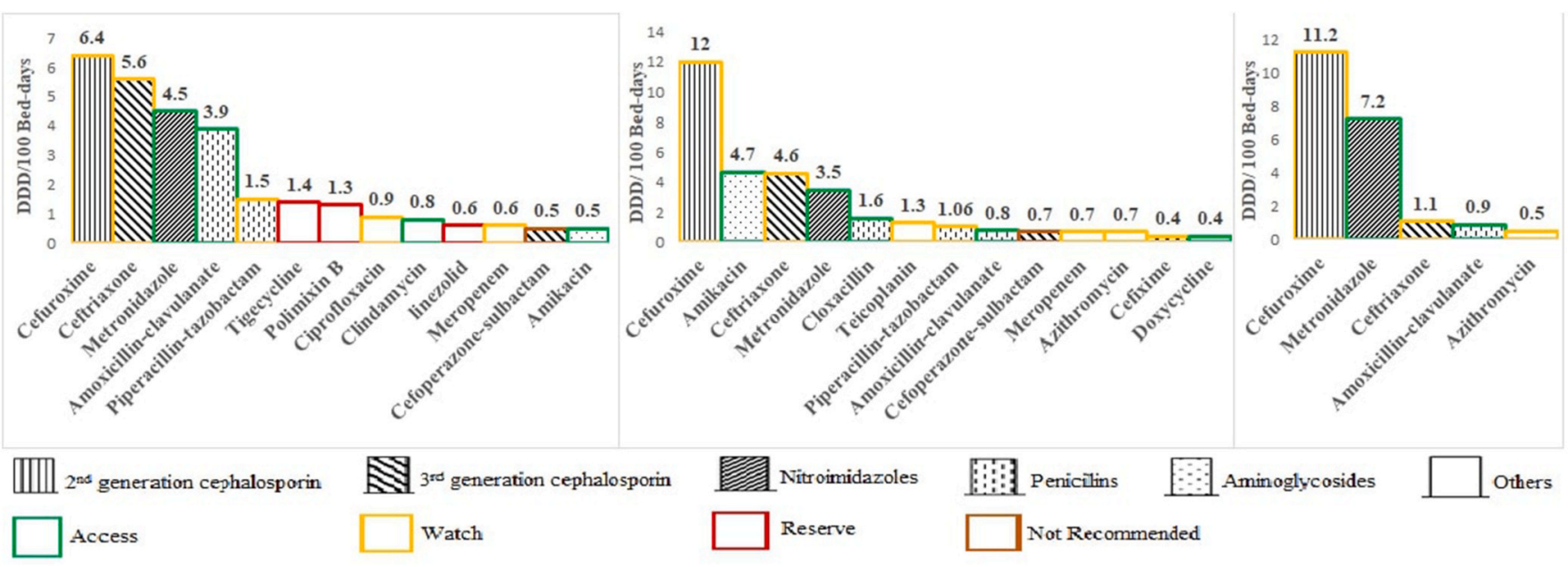

Fig. 1. Drug utilization 90\% (DU90\%) profiles of antibiotics using WHO AWaRe classification: (a) Surgery, (b) Orthopedics, (c) Gynecology, DDD: Defined Daily Dose.

Table 4

Compliance of antibiotic prescriptions and non-compliance parameters within various wards towards hospital antibiotic policy.

\begin{tabular}{|c|c|c|c|c|}
\hline & Surgery & Orthopedics & Gynecology & Total \\
\hline Total Compliance & $\begin{array}{l}1688 \\
(67.9 \%)\end{array}$ & $730(86.3 \%)$ & $\begin{array}{l}1365 \\
(88.6 \%)\end{array}$ & $\begin{array}{l}3783 \\
(77.7 \%)\end{array}$ \\
\hline \multicolumn{5}{|c|}{ Parameters of Non- compliance to hospital antibiotic policy } \\
\hline Duration & $489(19.7 \%)$ & $73(8.6 \%)$ & $163(10.6 \%)$ & $725(14.8 \%)$ \\
\hline Indication & $299(12 \%)$ & $40(4.7 \%)$ & $8(0.5 \%)$ & $347(7.1 \%)$ \\
\hline Dose/Route & $3(0.1 \%)$ & $1(0.2 \%)$ & $4(0.3 \%)$ & $8(0.2 \%)$ \\
\hline Wrong choice & $7(0.3 \%)$ & $1(0.2 \%)$ & & $8(0.2 \%)$ \\
\hline $\begin{array}{l}\text { Total } \\
\text { Prescriptions }\end{array}$ & 2486 & 845 & 1540 & $\begin{array}{l}4871 \\
(100 \%)\end{array}$ \\
\hline
\end{tabular}

cefazolin availability and concerns about antibiotic resistance.

The rate of empiric antibiotic use in our study was higher $(24.8 \%)$ compared to a European study (17\%), due to adherence to hospital guidelines. ${ }^{14}$ In $36.1 \%$ patients in our study, empirical therapy was not de-escalated after receiving culture reports due to concerns of infection in patients with immune-compromised states. Compared to our previous study $^{5}$ in the surgery ward, the empiric use of antibiotics has been reduced to $28.9 \%$ from $61 \%$. The antibiotic consumption shows cephalosporin as the preferred antimicrobial, with second generation cephalosporins being more commonly prescribed than first and third generations, which contradicts our previous study. ${ }^{5}$ This positive shift in prescribing behavior can be attributed to the implementation of our hospital's antibiotic policy and frequent audits by clinical pharmacist. Our hospital's antibiotic policy recommends cefuroxime/cefazolin as surgical prophylaxis for all surgeries and cefuroxime with metronidazole for abdominal surgeries. ${ }^{7}$ The third most commonly prescribed agent was ceftriaxone. This could be attributed to its success in controlling and treating infections due to its broad spectrum of activity against most bacterial species, particularly gram-negative microorganisms, as well as its widespread availability in hospital settings. ${ }^{15}$ However, in other Indian studies, higher prescription rates of ceftriaxone use was observed. ${ }^{5,16}$ The use of third generation cephalosporins must be monitored because they have been linked to the emergence of AMR. ${ }^{17}$

According to WHO AWaRe policy, ${ }^{18}$ the proportion of Access antibiotics should be greater than $60 \%$ of total antibiotic use. However, in our study, $53 \%$ of antibiotics were prescribed from the Watch group, while only $36 \%$ were prescribed from the Access group (Fig. 2). The reasons were use of cefuroxime instead of cefazolin for SP, use of empiric therapy without indication $(7.1 \%)$ and the concerns about treatment failure caused by antimicrobial resistance to WHO's Access group of

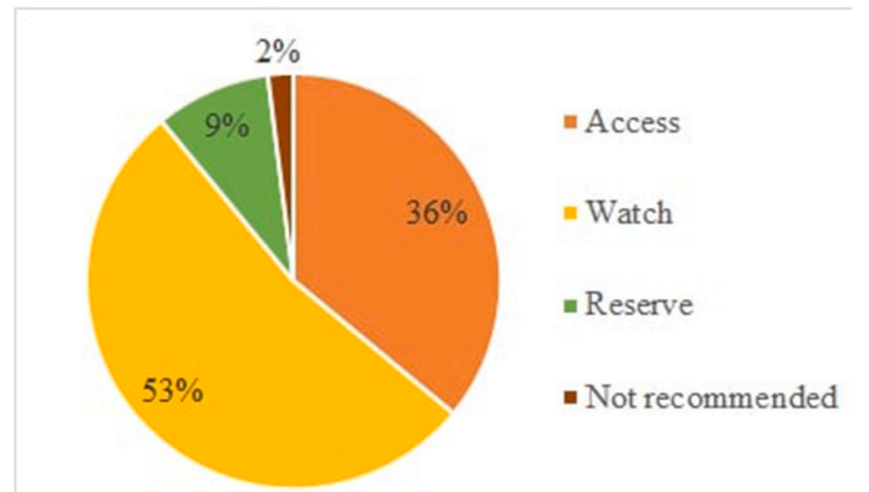

Fig. 2. Percentage of antibiotic use according to WHO AWaRe classification.

antibiotics. ${ }^{19}$ Hence, there is a need for local and country-specific modifications to the WHO AWaRe classification due to differences in AMR, antibiotic use, and treatment guidelines, in order to ensure it is optimized for country-specific use. Furthermore, the use of 'not recommended' antibiotic combinations was observed in our study, owing to a perceived positive clinical effect with their use. A national study conducted in India revealed the proliferation of unapproved antibiotic combinations as a barrier to controlling AMR in the country. ${ }^{20}$

The current study found that the number of antimicrobial agents in the DU90\% segment ranged from 5 to 13 , which is greater than the DU90\% profiles seen in other Indian (11 agents) and Ethiopian hospitals (6-8 agents). ${ }^{21,22}$ The DU90\% technique assumes that by employing a small number of different items, prescribing quality would be better. ${ }^{11}$

The compliance rate of antibiotic prescribing to hospital antibiotic policy has improved to $67.9 \%$ compared to our previous study $(27.2 \%)$ in the surgery ward. ${ }^{5}$ Similar, compliance rate $(70 \%)$ was reported in surgery wards of a study conducted in India. ${ }^{12}$ Among patients who received antibiotics for surgical prophylaxis, $22.5 \%$ patients were treated for more than $24 \mathrm{~h}$, which contradicted the recommendations of the hospital antibiotic policy and this needs attention. The extended duration of SP could be due to the widespread belief among physicians that it can prevent surgical site infections (SSIs) in surgical wound cases with a high risk of bacterial contamination. Prolonged SP is generally unnecessary, and it raises the risk of AMR and side effects. ${ }^{11,12}$ The hospital antibiotic policy requires appropriate cultures to be sent prior to the start of empiric therapy, which was not followed [54.7\% of patients received more than 3 days of empiric therapy]. According to a systematic review and meta-analysis, guideline-compliant empirical 
therapy was associated with a $35 \%$ reduction in relative risk of mortality. ${ }^{23}$ Prolonged SP or definitive therapy, empiric therapy without appropriate microbiological cultures, and noncompliance with hospital antibiotic policy can all lead to increased antibiotic prescribing and, as a result, an increase in antibiotic resistance. ${ }^{11,12,23}$

The study's strength is its large sample size, which allows for a diverse range of infections to be included in order to answer the research questions. The study makes several important recommendations to improve future antibiotic use. The study's limitations include the lack of a hospital information system for rapid data collection and tracking of antimicrobial prescriptions, as well as the lack of a single point of contact for antimicrobials prescribed in the hospital. As a result, a costbenefit analysis of antimicrobial consumption could not be performed.

\section{Conclusion}

The hospital's antibiotic policy was followed with a high degree of adherence. However, antibiotic prescriptions without indication, surgical prophylaxis for longer than necessary, and empiric therapy without microbiological culture reports all contribute to noncompliance with the hospital antibiotic policy. In comparison to access antibiotics, Watch group antibiotics are used more frequently. This highlights the need of antibiotic surveillance programs and further development of antibiotic treatment guidelines based on WHO AWaRe policy.

\section{Funding}

This research received no specific grant from any funding agency in the public, commercial, or not-for-profit sectors.

\section{Ethical approval}

Obtained.

\section{Declaration of competing interest}

The authors declare that they have no competing interests.

\section{References}

1 Van Boeckel TP, Gandra S, Ashok A, et al. Global antibiotic consumption 2000 to 2010: an analysis of national pharmaceutical sales data. Lancet Infect Dis. 2014;14(8): $742-750$.

2 Klein Eili Y, Milkowska-Shibata Maja, Tseng Katie K, et al. Assessment of WHO antibiotic consumption and access targets in 76 countries, 2000-15: an analysis of pharmaceutical sales data. Lancet Infect Dis. 2020;21(1):107-115.

3 Bell BG, Schellevis F, Stobberingh E, Goossens H, Pringle M. A systematic review and meta-analysis of the effects of antibiotic consumption on antibiotic resistance. BMC Infect Dis. 2014;14(13):1-25.
4 Olesen SW, Barnett ML, MacFadden DR, et al. The distribution of antibiotic use and its association with antibiotic resistance. Elife. 2018;18(7), e39435.

5 Soman N, Panda BK, Banerjee JK, John SM. A study on prescribing pattern of cephalosporins utilization and its compliance towards the hospital antibiotic policy in surgery ward of a tertiary care teaching hospital in India. Int Surg J. 2019;6(10): 3614.

6 Senthilkumar S, SA AR, Padmavathi K, Dhanapal CK, Periasamy K. Study on antibiotic use among geriatric patients based on anatomical therapeutic classification or defined daily dose methodology and world health organization-essential medicine list access, watch and reserve concept in tertiary care hospital of South India. Int J Basic Clin Pharmacol. 2020;9(7):1106-1113.

7 Bharati Hospital and Research Center Antibitoic Policy Version 5.0. 2019 [Available from: Microsoft Word - ANTIBIOTIC POLICY UPDATED BHARATI HOSPITAL 2019 Version 18.02.2020 (bharatividyapeeth.edu)].

8 Demeke B, Molla F, Assen A, et al. Evaluation of drugs utilization pattern using who prescribing indicators in Ayder referral hospital, Northern Ethiopia. Int J Pharma Sci Res. 2015;6(2):343-347.

9 Sataloff RT, Johns MM, Kost KM. Guidelines for ATC Classification and DDD Assignment. WHO; 2020 [Availabe from: 2020_guidelines_web-ATC-OMS.pdf (udec. cl)].

10 Zhang W, Shen X, Bergman U, et al. Drug utilisation $90 \%$ ( DU90 \%) profiles of antibiotics in five Chinese children' s hospitals ( 2002 - 2006 ). Int J Antimicrob Agents. 2008;32(3):250-255.

11 Versporten A, Zarb P, Caniaux I, et al. Antimicrobial consumption and resistance in adult hospital inpatients in 53 countries: results of an internet-based global point prevalence survey. Lancet Global Health. 2018;6(6):e619-e629.

12 Singh SK, Sengupta S, Antony R, et al. Variations in antibiotic use across India: multicentre study through Global Point Prevalence survey. J Hosp Infect. 2019;103(3): 280-283.

13 World Health Organization. Global guidelines for the prevention of surgical site infection. Available from http://apps.who.int/iris/bitstream/10665/250680/1/9 789241549882-eng.pdf?ua=1; 2016.

14 Kumarasamy Y, Cadwgan T, Gillanders IA, Jappy B, Laing R, Gould IM. Optimizing antibiotic therapy - the Aberdeen experience. Clin Microbiol Infect. 2003;9(5): 406-411.

15 Pinto Pereira LM, Phillips M, Ramlal H, Teemul K, Prabhakar P. Third generation cephalosporin use in a tertiary hospital in Port of Spain, Trinidad: need for an antibiotic policy. BMC Infect Dis. 2004;4(1):59.

16 Sane RM, Shahani SR, Kalyanshetti AA. Antibiotic prescription pattern in surgical wards of MGM hospital. Kamothe. Int J Infect. 2018;5(1), e57914.

17 Park SH. Third-generation cephalosporin resistance in gram-negative bacteria in the community: a growing public health concern. Korean J Intern Med. 2014;29(1): $27-30$.

18 World Health Organization. AWaRe policy brief [Available from: https://adoptaware .org/assets/pdf/aware_policy_brief.pdf; 2019.

19 Gandra S, Kotwani A. Need to improve availability of 'access' group antibiotics and reduce the use of 'watch' group antibiotics in India for optimum use of antibiotics to contain antimicrobial resistance. J Pharm Policy Pract. 2019;12(1):20-23.

20 Mcgettigan P, Roderick P, Kadam A, Pollock AM. Reserve antibiotics in India : challenges for WHO stewardship. Lancet Global Health. 2011;5(11):e1075-e1076.

21 Bhardwaj A, Kapoor Kaveri, Singh Vivek. Trend analysis of antibiotics consumption using WHO AWaRe classification in tertiary care hospital. Int J Basic Clin Pharmacol. 2020;9(11):1675-1680.

22 Gutema Girma, Håkonsen Helle, Engidawork Ephrem, Toverud Else-Lydia. Multiple challenges of antibiotic use in Ethiopia - a ward-specific study showing high rates of hospital-acquired infections and ineffective prophylaxis. BMC Health Serv Res. 2018; 18(1):326.

23 Schuts EC, Hulscher M, Mouton JW, et al. Current evidence on hospital antimicrobial stewardship objectives: a systematic review and meta-analysis. Lancet Infect Dis. 2016;16(7):847-856. 